Check for updates

Cite this: J. Mater. Chem. C, 2019, 7, 5104

Received 30th December 2018 Accepted 25th February 2019

DOI: $10.1039 / \mathrm{c} 8 \mathrm{tc} 06621 \mathrm{c}$

rsc.li/materials-c

\section{Improved photostability in ternary blend organic solar cells: the role of [70]PCBM $\dagger$}

\author{
Nutifafa Y. Doumon, (D)*a Félix V. Houard, (D) ${ }^{a}$ Jingjin Dong, ${ }^{b}$ \\ Panagiotis Christodoulis, ${ }^{a}$ Mikhail V. Dryzhov, ${ }^{a}$ Giuseppe Portale (D) ${ }^{b}$ and \\ L. Jan Anton Koster (D) *a
}

\begin{abstract}
Polymer solar cells are potentially key contributors to the next-generation organic photovoltaics for sustainable green sources of energy. In the past few years, ternary organic solar cells have emerged with promising characteristics. They have proven to yield high efficiency at about $15 \%$ for single junction donor:acceptor (D:A) solar cells. However, the low stability of organic solar cells is a hindrance to the commercialisation of this technology, and thus, needs more attention. Here, we show that with the right ratio of $D: A_{1}: A_{2}$, ternary blend solar cells can be more efficient and more photostable than their D:A binary blend solar cells. We add [70]PCBM to PBDB-T:ITIC and PTB7-Th:ITIC binary blend solar cells in various ratios to fabricate ternary solar cells. The ternary solar cells outperform all binary cells in terms of efficiency and photostability with only a $10 \%$ average loss in efficiency under continuous illumination irrespective of the device structure. We identify changes in the molecular structure of the active layer blends as the main reason behind the observed degradation behaviour of the solar cells. The ternary blends are the most resilient to photo-induced molecular structural changes. This finding suggests that ternary organic solar cells could be a way to achieve photostable devices.
\end{abstract}

\section{Introduction}

Bulk heterojunction (BHJ) organic solar cells (OSCs) have attracted much attention in the past decades as they offer the possibility to achieve high power conversion efficiency (PCE). This is due to the opportunities offered for easy processing of the active layer namely the ability for solution processing and the mixture of (novel) organic materials readily soluble in various solvents. ${ }^{1}$ The latter brought into conception the ternary blend OSCs. The ternary blend OSCs differ from the well-known binary blend OSCs by one additional component in the mixture of their active layer organic materials. This means one can migrate from a donor:acceptor (D:A) binary system to either a D:A:A ${ }^{2-5}$ or a D:D:A $A^{6-9}$ ternary system. The ternary blends have helped achieve over the last few years a PCE of more than $14 \%{ }^{10-14}$ in single junction OSCs and lately a record $17.3 \%{ }^{15}$ in tandem structure

\footnotetext{
${ }^{a}$ Photophysics and Optoelectronics, Zernike Institute for Advanced Materials, University of Groningen, Nijenborgh 4, NL-9747 AG, Groningen, The Netherlands. E-mail:n.y.doumon@rug.nl, l.j.a.koster@rug.nl

${ }^{b}$ Macromolecular Chemistry and New Polymeric Materials, Zernike Institute for Advanced Materials, University of Groningen, Nijenborgh 4, NL-9747 AG, Groningen, The Netherlands

$\dagger$ Electronic supplementary information (ESI) available: Time evolution of $J-V$ parameters of the studied solar cells, $J-V$ parameters of the studied solar cells, ratio-dependent photovoltaic performance of PBDB-T based solar cells, light intensity dependence measurements, and GIWAXS peak intensity plots. See DOI: $10.1039 / \mathrm{c} 8 \mathrm{tc} 06621 \mathrm{c}$
}

serving as the rear sub-cell. This is because of the flexibility in choices of the additional $\mathrm{D}$ or A material such that a wider range of the solar spectrum is covered due to the complementarity of the additional material absorption profile to the parent cell's active layer absorption spectrum. The result could be an extension to the near infrared region or an improvement in the absorption strength for shorter wavelengths. ${ }^{16,17}$ The additional component serves as a bridge in the energy levels of the parent materials and creates an energetic cascade effect in the ternary blends by either facilitating charge transport as a charge relay component or as an agent of energy transfer, an antenna, and thus, improving the overall cell performance. ${ }^{17}$ This improvement in PCE is not only limited to these electronic effects. There could also be a positive structural effect. Thus, the third component could also enhance the nanomorphology $y^{3,18-20}$ of the active layer to help in better charge dissociation and transport. However, this is not always the case; the addition of a third component could also negatively affect the nanostructure of the blend. Therefore, there should be a trade-off in the ratio of the three components depending on the materials used. A third proposed working mechanism of the ternary blend is termed the parallel-like model, in which the device works as two intermixed binary or tandem like solar cell. This working mechanism is also called the alloy model, in which it is thought that either the donors or the acceptors form a kind of electronic alloy. Thus, the new HOMO and LUMO levels are considered to be the average of the individual energy levels. It is suggested that in the alloy concept, the $V_{\text {oc }}$ of the ternary device 
linearly scales with the blend ratios as opposed to pinning it to the difference in energy levels of the $D$ and $A,{ }^{17,21-24}$ a point of view which is still under debate in the literature. The studies in the performance of the ternary blend solar cells have so far been only limited to the PCE. Then again, the same is true for the new class of binary OSCs, the non-fullerene solar cells. ${ }^{8,25-27}$ Only a few studies have truly tried to understand the stability issues related to these types of OSCs, and thus, study the degradation mechanisms in such cells. ${ }^{2,28,29}$ Few reports suggested the non-fullerene OSCs are relatively more stable than their fullerene counterparts, but no comparative studies have been carried out under the same conditions. $^{30-32}$

Here, we propose a systematic study under the same experimental and environmental conditions into the photostability of two binary OSCs namely, fullerene and non-fullerene OSCs, and $\mathrm{D}: \mathrm{A}_{1}: \mathrm{A}_{2}$ ternary OSCs. The $\mathrm{A}_{1}: \mathrm{A}_{2}$ component of the ternary OSC is made up with the same fullerene and non-fullerene acceptors of the binary blends. Our study mainly employed the workhorse polymer PBDB-T as the donor material, [70]PCBM and ITIC as the fullerene and non-fullerene acceptor materials respectively, as shown in Fig. 1. To ascertain our finding, PTB7-Th workhorse low bandgap polymer ${ }^{33-35}$ was also employed in place of PBDB-T. Under the same experimental conditions, the ternary blend OSCs outperform by far the [70]PCBM binary blend OSC in terms of PCE and are slightly better in PCE than the ITIC binary blend OSC. More interestingly, we show that the ternary solar cells are the most efficient, followed by ITIC binary and then [70]PCBM binary solar cells. It is equally the most photostable over the period of the study, followed by [70]PCBM binary and then ITIC binary solar cells. This makes the non-fullerene binary OSCs the least photostable in the series. Additionally, through experimental techniques such as absorption spectroscopy, atomic force microscopy (AFM), grazing incidence wideangle X-ray scattering (GIWAXS), and charge transport studies we explain the reasons behind the observed trend, thus, a decrease in photostability under continuous illumination: from the ternary blend to the fullerene binary blend with the least photostable being the non-fullerene binary blend solar cells. This study suggests that ternary blends could be a way to more efficient as well as photostable OSCs. Thus, more attention is to be invested in studies in this direction to ascertain this claim.

\section{Results \& discussion}

We fabricated both conventional and inverted structure OSCs of binary blends on the one hand and ternary blends on the other hand by varying the acceptor ratio $\left(A_{1}: A_{2}\right)$ from 0 to 1 for PBDBT-based blends (or $0: 1.5$ for PTB7-Th-based blends) in a ratio of $1: 1$ dissolved in anhydrous chlorobenzene (CB) at a concentration of $20 \mathrm{mg} \mathrm{mL}^{-1}$. The cells are exposed continuously to 1 sun illumination for two hours at open-circuit, keeping them at room temperature in an inert atmosphere.

Fig. 2a reveals the average degradation pathway of the studied conventional OSCs (minimum of 10 devices) for their photostability while Fig. 2c shows the photodegradation behaviour of the inverted cells (minimum of 10 devices). The PBDB-T:ITIC binary blend OSCs suffer the most in degradation with more than $20 \%$ efficiency loss after 2 hours of illumination, regardless of the configuration $(-23 \%$ for the conventional OSCs, and $-20 \%$ for the
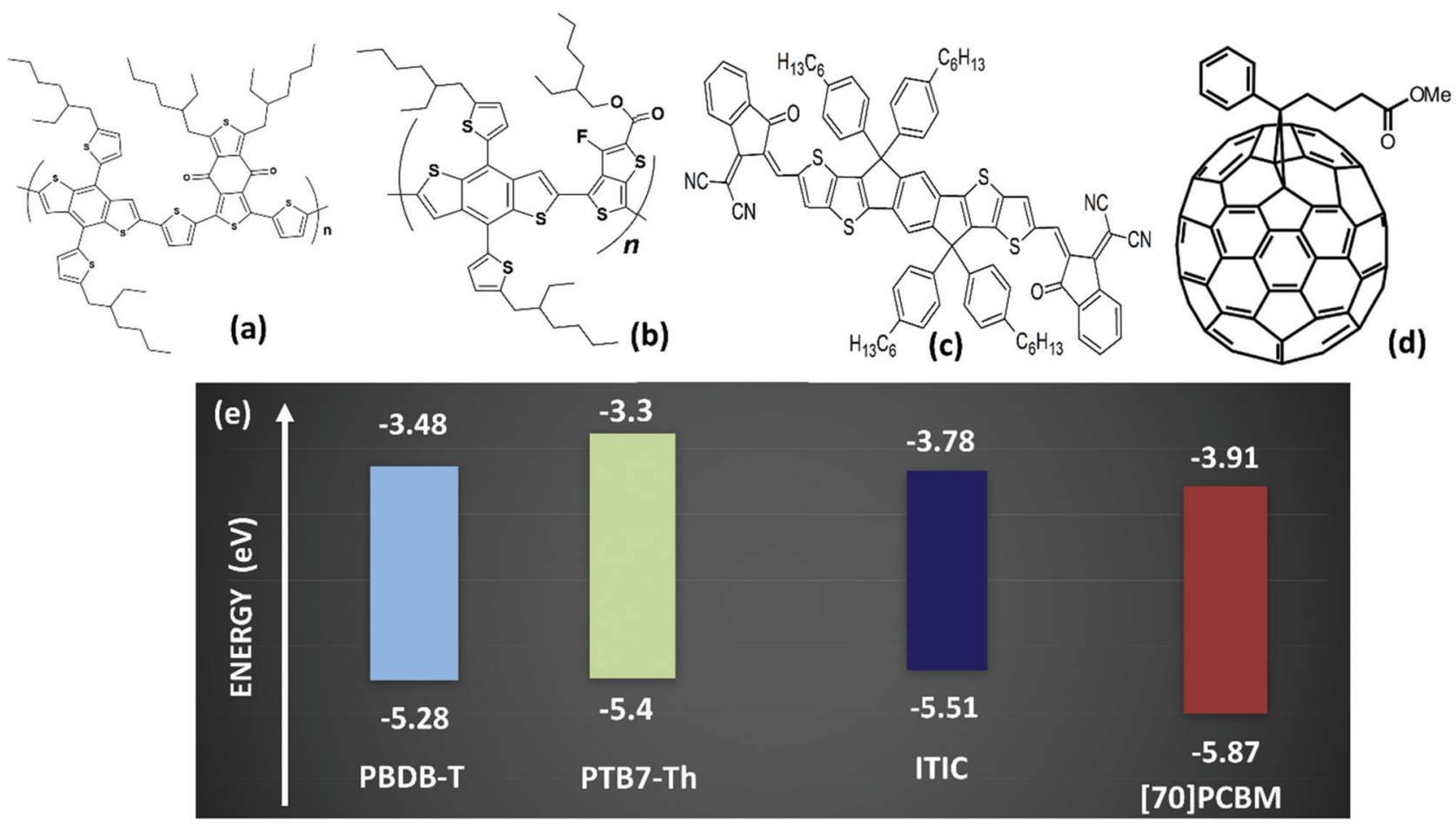

Fig. 1 Semiconductor materials employed: PBDB-T (a), PTB7-Th (b), ITIC (c), [70]PCBM (d) with their corresponding HOMO-LUMO energy levels (e). 

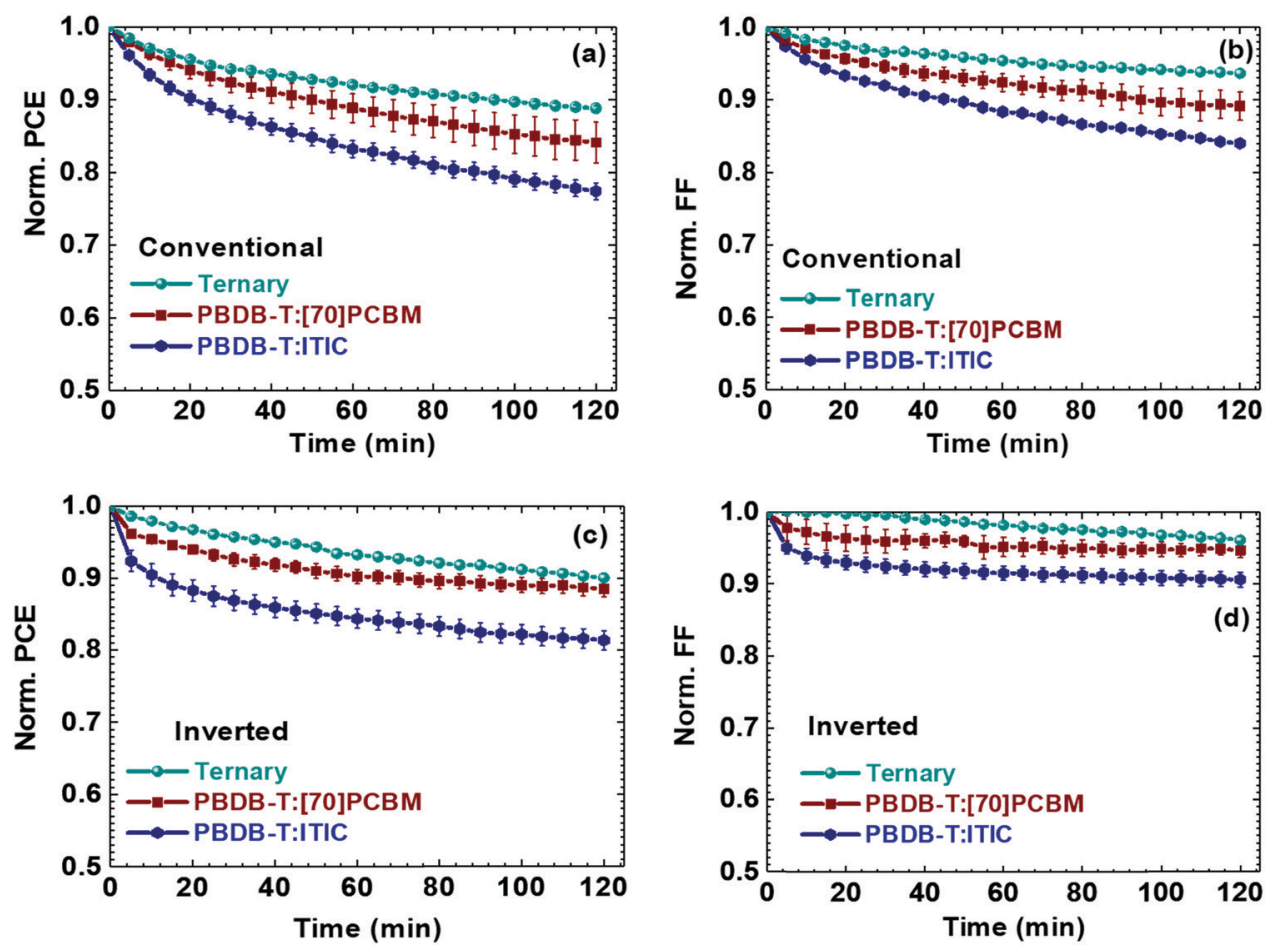

Fig. 2 Photo-induced degradation behaviour of PCE ( $a$ and $c$ ) and FF ( $b$ and d) of the conventional ( $a$ and b) and inverted (c and d) solar cells: ternary (sphere, cyan), PBDB-T:[70]PCBM (square, brown) and PBDB-T:ITIC (circle, blue).

inverted ones). In contrast, [70]PCBM-based devices take advantage of the inverted configuration, with an enhancement in stability from $-16 \%$ to $-12 \%$. The most stable blend is the ternary blend with a limited degradation rate around $-10 \%$ for both conventional and inverted configurations. Clearly, the ternary blend solar cells are more photostable than the binary blend solar cells.

Fig. 2b, d, and Fig. S1 (ESI $\dagger$ ) display the corresponding degradation behaviour of the rest of the $J-V$ parameters. Generally, as observed in Fig. S1 (ESI $\dagger$ ) the $J_{\text {sc }}$ and $V_{\text {oc }}$ remain fairly constant, excluding a potential photo-bleaching phenomenon (a loss in absorption) or changes in the HOMO and LUMO energy levels. However, the FF, as seen in Fig. $2 \mathrm{~b}$ and d show the largest loss, and thus, remains the main contributor to the observed PCE loss in all three devices. Graphically, the loss behaviour of the PCEs is very similar to that of the FFs. The most significant finding is that the ternary blend solar cells are substantially more photostable than the ITIC based binary solar cells. To ascertain this finding, PBDB-T is substituted with PTB7-Th, and the experiments are repeated for two best ratios of the ternary blends. Fig. S2 (ESI $\dagger$ ) distinctively displays the PCE decay behaviour of the single devices. Fig. S2c and $\mathrm{d}$ (ESI $\dagger$ ) show clearly that the observation is true for both polymers and the different ratios. It is important to note that the degradation trends of the three solar cells are preserved irrespective of the device configuration, which allows excluding a possible impact of the different interfacial layers such as PEDOT:PSS, ZnO, $\mathrm{MoO}_{3}$, or LiF on the evolution of the photovoltaic performances over time. These changes are hence inherent to the active layer. The single degradation plots of the devices, conventional and inverted, in Fig. S2a and b (ESI $\dagger$ ) reveal the real degradation behaviour of the three types of solar. The binary devices show the "burn-in" degradation behaviour known for solar cells under continuous illumination while the ternary ones only show a steady, gradual degradation behaviour. These findings do not imply that there is photostability beyond a couple of hours. We are limited within 2 hours in our study as we wanted to absolutely make sure that the devices are measured under the same conditions. Thus, more testing is needed to establish the long-term behaviour. However, there is a clear difference in the first two hours.

Additionally, the ternary blends outperform the binary blends in terms of PCE. Table 1 shows the $J-V$ parameters of the selected OSCs in each category, fabricated under the same conditions, while Fig. S3 (ESI $\dagger$ ) displays the $J-V$ curves of the best performing OSCs, their external quantum efficiency (EQE), the absorption spectra of their films, and the device statistics. Before conducting the photodegradation experiments on the studied OSCs, we first work out the ratio of the binary and ternary blends in order to have them practically under the same conditions. Previously, the ratio of $1: 1$ has been reported to be the best for PBDB-T: ITIC binary devices. To have the same concentration for all blend solutions, the same ratio was used to gauge the ternary blends. While keeping constant the ratio of PBDB-T in the ternary blends, the ratio of the $A_{1}: A_{2}$ part of the $D: A_{1}: A_{2}\left([D]:\left[A_{1}\right]:\left[A_{2}\right]=1:\left[A_{1}\right]: 1-\left[A_{1}\right]\right)$ is changed to optimize efficiency and stability. Tables S1 and S2 (ESI $\dagger$ ) show the full range of the ratios covered for the conventional ternary blend OSCs as an example while Fig. S4 (ESI $\dagger$ ) shows the dependency of the $J-V$ parameters on the blend ratios. It is clear that our ternary blend devices do not conform with the alloy model ${ }^{22-24}$ as the $V_{\mathrm{oc}}$ shows no linear dependency on the blending ratio, as is the case for the rest of the parameters. From Table S1 (ESI + ), there 
Table $1 J-V$ parameters of solar cells made under the same conditions for comparison: conventional (conv.) and inverted (inv., italic) where mn: mean and SD: standard deviation. Full ratio details of conv. solar cells can be seen in Tables S1 and S2 (ESI)

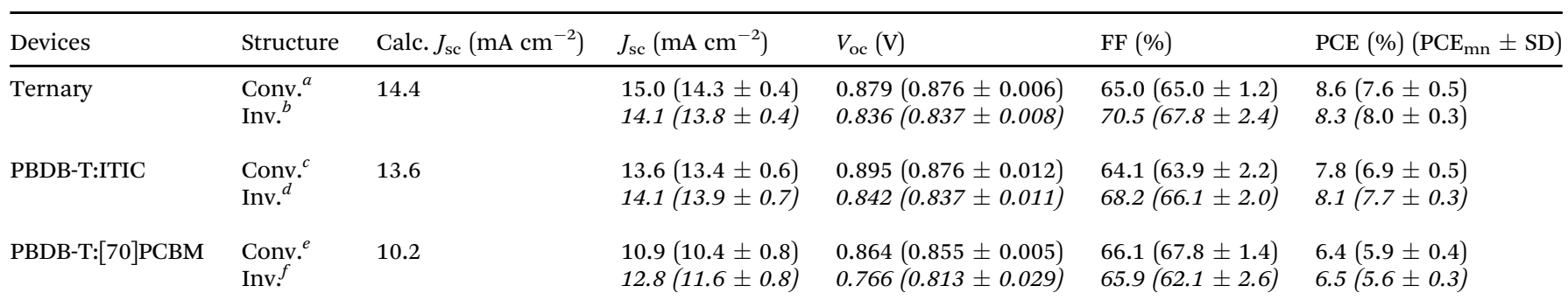

${ }^{a} 27$ devices. ${ }^{b} 11$ devices. ${ }^{c} 13$ devices. ${ }^{d} 18$ devices. ${ }^{e} 10$ devices. ${ }^{f} 19$ devices.

are three ratios of PBDB-T: ITIC:[70]PCBM that recorded similar but highest PCEs, namely $1: 0.9: 0.1,1: 0.8: 0.2$ and $1: 0.7: 0.3$. Thus, the photodegradation experiments are primarily conducted for these ratios together with the binary blends. Fig. S2c (ESI $\dagger)$ shows this primary result, revealing similar photo-degradation behaviour for all three ratios with $1: 0.8: 0.2$ and $1: 0.7: 0.3$ being slightly better. Our study mainly considered the $1: 0.7: 0.3$ ratio for PBDB-T:ITIC:[70]PCBM as it yielded the best average PCE. Very recently, it was found by Wang et al. that the addition of [70]PCBM as a third component in PBDB-T:ITIC binary cells improves the efficiency, with $1: 0.8: 0.2$ ratio yielding the best efficiency in their device structure, ${ }^{36}$ as is the case also in our study (see Table S1, ESI $\dagger$ ). Here, we show that [70]PCBM does not only improve the PCE of PBDB-T:ITIC binary cells, but also that of PTB7-Th:ITIC binary cells (with ratios of $1: 0.5: 0.5$ and $1: 0.75: 0.75$ ) as well as the photostability of the parent binary cells.

The superior performance of the ternary blends in terms of PCE has been sparingly touted in the literature. However, the reason why they perform better in terms of photodegradation than the binary blends has not been thoroughly investigated and remained largely unclear. Indeed, ternary blend solar cells lead to high efficiency in few cases, ${ }^{12,15}$ but in general, the efficiency of these cells are largely poor. We believe it is in the interest of the scientific community to study the few that show promising results so to understand their working mechanisms. It is in this need that we set out to investigate the aforementioned observed behaviours by studying all three blends in details: PBDB-T:ITIC, PBDB-T:[70]PCBM and PBDB-T: ITIC:[70]PCBM. We perform a series of experiments including UV-vis absorption spectroscopy and atomic force microscopy on their respective films before (fresh) and after exposure (exposed) to simulated sunlight for 2 hours, under the same condition as the solar cells. The absorption spectra of the fresh films are displayed in Fig. S3b (ESI $\dagger$ ). Just as depicted in the EQE spectra in Fig. S3c (ESI $\dagger$ ) the ternary film shows a slight improvement at lower wavelengths compared to the ITIC binary film while displaying similar contribution as ITIC binary at the longer wavelengths. Also, the AFM images show the ternary films as an intermediate morphology with the mean roughness of $2.8 \mathrm{~nm}$ between the ITIC binary with the roughest morphology $(3.6 \mathrm{~nm})$ and the [70]PCBM binary with the smoothest morphology $(1.2 \mathrm{~nm})$. This may explain in part the better performance of the ternary cells as they benefit from enhanced nanomorphology ${ }^{3,18}$ as compared to the ITIC binary blends. However, when we compare the absorption spectra of the fresh to the exposed films in Fig. 3a-c, no significant changes were detected as earlier speculated to suggest photo-bleaching as the cause of the degradation. The AFM images of the fresh and exposed films in Fig. 3d-f also show no significant changes capable of explaining the observed photodegradation behaviour of these blends over the period of exposure, though morphological changes cannot be totally discounted.

Next, we performed $J_{\mathrm{sc}}$ and $V_{\mathrm{oc}}$ light intensity dependence measurements on the fresh and exposed $(1 \mathrm{~h}$ and $2 \mathrm{~h})$ solar cells to monitor the changes in recombination within the device. The presence of bimolecular recombination is highlighted by a sublinear behaviour of the $J_{\mathrm{sc}}$ with the light intensity. Thus, $J_{\mathrm{sc}}$ is proportional to $I^{\alpha}$ with $\alpha$ below or close to unity. No changes in $\alpha$ values are observed before and after exposure for the PBDB-T:ITIC $(\alpha=0.96)$, PBDB-T:[70]PCBM $(\alpha=0.97)$ and the ternary blends $(\alpha=0.96)$ in Fig. S4a-c (ESI $\dagger)$. In addition to bimolecular recombination, trap-assisted recombination also contributes to losses in solar cells, as highlighted by the calculated ideality factors, $n$, above unity. It is especially the case for the fullerene blend solar cell, which exhibits a slightly higher density of traps with its $n$ of 1.5 , more than the 1.2 for the ITIC blend, and 1.29 for the ternary blend solar cells. Fig. S4d-f (ESI $\dagger$ ) shows the variation of the $V_{\text {oc }}$ with light intensity. We can observe that the $V_{\text {oc }}$ of the fresh ternary blend device is pinned around that of the ITIC binary device. The extracted $n$ from Fig. S4d-f (ESI $\dagger$ ) reveal no change in $n$ for ITIC binary devices which remained constant at around 1.20 and only minute increases in $n$ for the [70]PCBM binary cells from 1.5 to 1.6 , and for the ternary devices from 1.29 to 1.33 . Thus, trap-assisted recombination cannot be ascribed to the observed degradation pattern; at least that is not the case for ITIC binary devices.

To elucidate the degradation process, we resort to studying the differences in molecular packing that may occur in the films at the fresh $(0 \mathrm{~h})$ and exposed (after $2 \mathrm{~h}$ and $4 \mathrm{~h}$ ) states. The structure of the PBDB-T:ITIC, PBDB-T:PCBM and PBDB-T: ITIC:PCBM films and their time-dependence behaviour during exposure are studied by grazing incidence wide-angle X-ray scattering (GIWAXS). Fig. 4 shows the GIWAXS patterns and Fig. S6 (ESI $\dagger$ ) their corresponding horizontal and vertical intensity cuts. As shown in Fig. 4, the image of PBDB-T:ITIC 

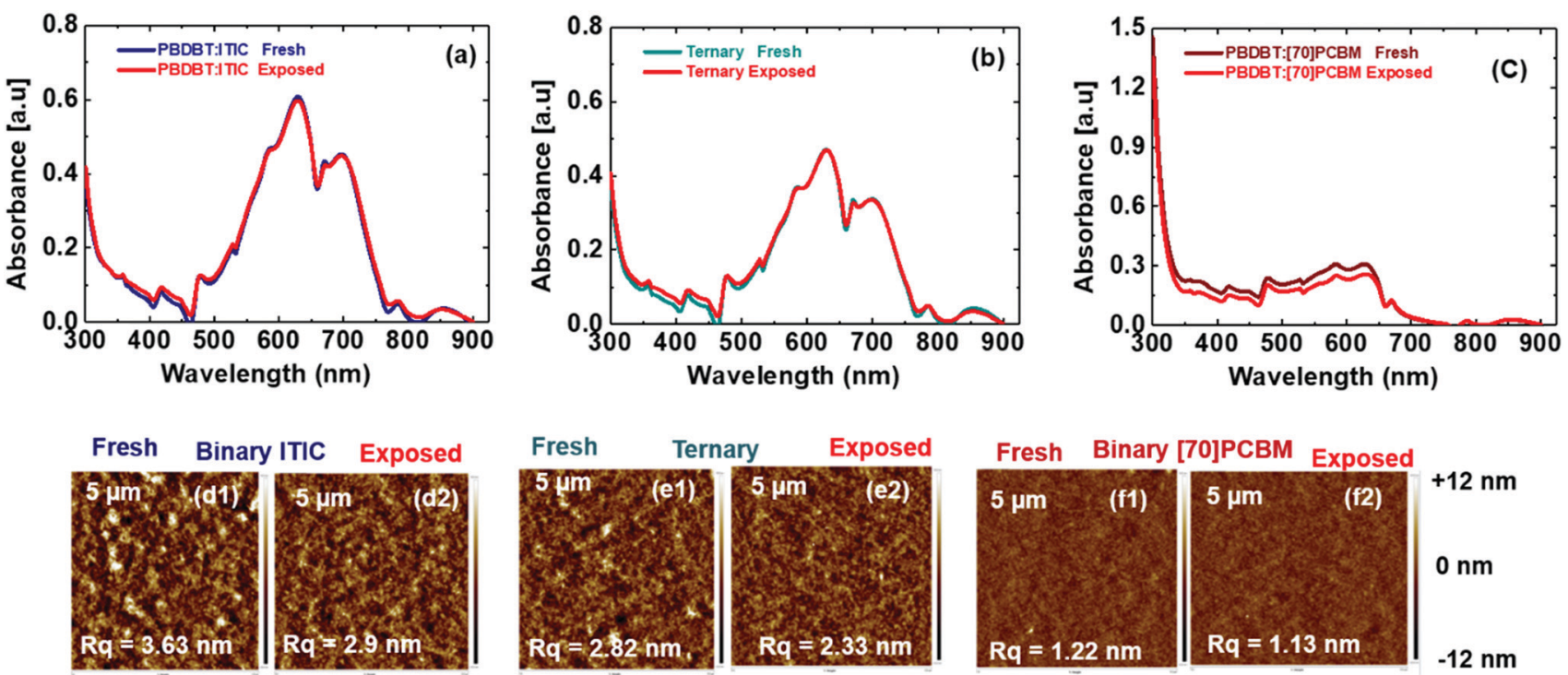

Fig. 3 Absorption spectra of fresh and exposed ( $2 \mathrm{~h}$ ) films: PBDB-T:ITIC (a), ternary (b), and PBDB-T:[70]PCBM (c). AFM images of fresh and exposed (2 h) films: PBDB-T:ITIC (d1 and d2), ternary (e1 and e2), and PBDB-T:[70]PCBM (f1 and f2).

fresh film shows an intense (010) peak located at $q_{z}=$ $17.1 \mathrm{~nm}^{-1}$, associated to a molecule $\pi-\pi$ stacking distance of $0.38 \mathrm{~nm}$ and concentrated along the vertical direction (see Fig. S6d, ESI $\dagger$ ), indicating a dominantly face-on orientation of the PBDB-T crystallites (see Fig. 4a). Conversely, the (010) peak of PBDB-T is not present in the GIWAXS pattern of the fresh PBDB-T:[70]PCBM film. Instead, the intensity of the (100) peak at $q_{z}=2.9 \mathrm{~nm}^{-1}$ is much higher than the PBDB-T:ITIC fresh film which means the PBDBT:[70]PCBM film is mainly packed as edge-on. The PBDBT:ITIC:[70]PCBM ternary blend fresh film shows a mixture of both binary structures; thus, a mixture of face-on and edge-on orientation.
The degradation upon light exposure was also assessed by GIWAXS. At different times during the exposure, the structural changes experienced by the three systems are quite different. Upon exposure, the (010) peak along the out-of-plane vertical direction of PBDB-T:ITIC film notably decreases over time together with a decrease of the (100) peak in the in-plain horizontal direction. This intensity decrease reported in Fig. S6a and d (ESI $\dagger$ ) suggests that the face-on packed crystallites significantly degrade and the molecular packing is negatively affected by light exposure. However, the edge-on packed crystallites also degraded dramatically as shown in the in-plane plots (see Fig. S6a, ESI†) of

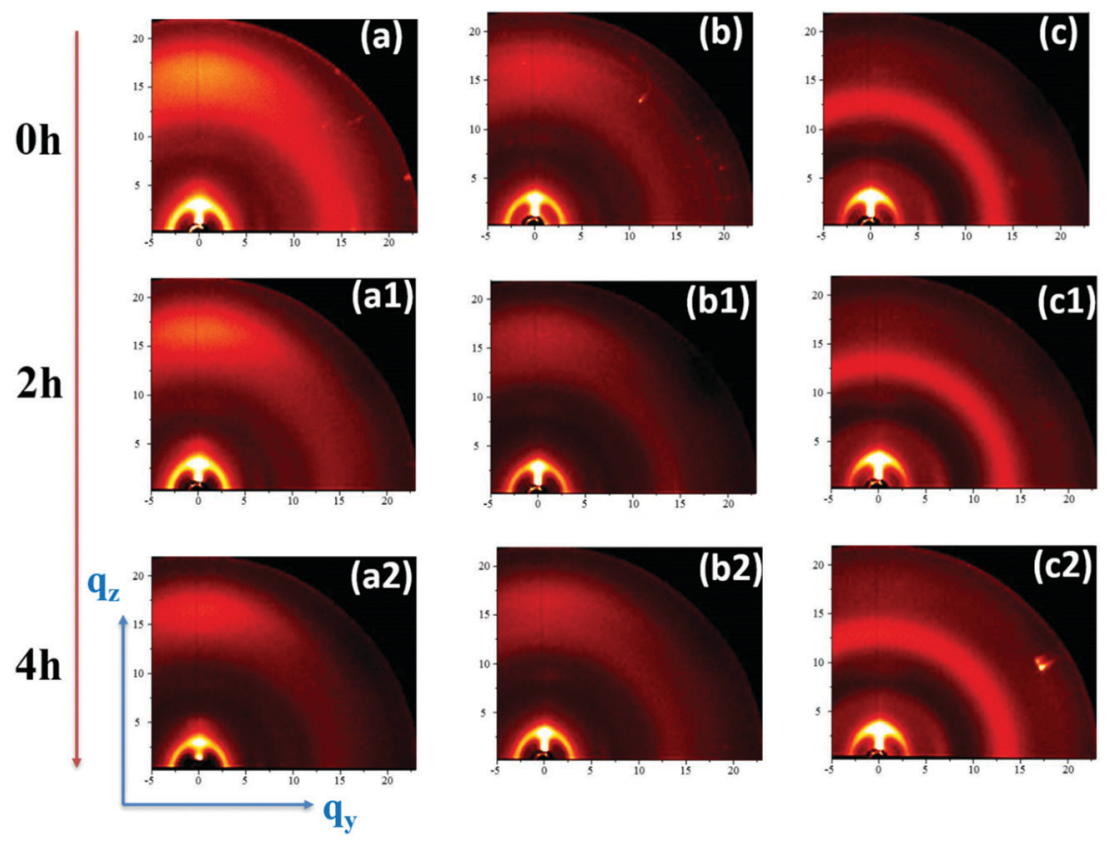

Fig. 4 GIWAXS images of fresh $(0 \mathrm{~h})$ and exposed $(2 \mathrm{~h}$ and $4 \mathrm{~h})$ films: PBDB-T:ITIC (a, a1, and a2), ternary (b, b1, and b2), and PBDB-T:[70]PCBM (c, c1, and $\mathrm{c} 2)$. Fresh $0 \mathrm{~h}(\mathrm{a}-\mathrm{c})$, exposed $2 \mathrm{~h}(\mathrm{a} 1, \mathrm{~b} 1$, and $\mathrm{c} 1)$ and exposed $4 \mathrm{~h}(\mathrm{a} 2, \mathrm{~b} 2$, and $\mathrm{c} 2)$. Note that the intensity scale is the same for all the patterns. 
PBDB-T: ITIC films with the (010) peak at $q_{y}=15.4 \mathrm{~nm}^{-1}$ disappearing after 2 hours and the out-of-plane (100) peak decreasing with time. The changes in the structure of the PBDB-T:ITIC films point clearly to a decrease in crystallinity and could impede charge mobility, creating unbalanced charges as already revealed in our previous study ${ }^{29}$ explaining why the FF dropped the fastest. Thus, the PBDB-T:ITIC binary cells recorded the fastest PCE decay among the three cells. In contrast, both the PBDB-T:[70]PCBM and the PBDB-T:ITIC:[70]PCBM ternary blend films are less affected by the exposure, with minimal structural changes upon exposure. This can explain the relatively good stability of these two blends with respect to the PBDB-T:ITIC binary solar cells. As stated earlier, the structural changes experienced by the PBDB-T:ITIC cells, linked to the unbalanced charge carrier mobilities in their active layers, is the main reason behind the losses observed in the ITIC binary devices. However, the [70]PCBM binary devices with their more balanced mobilities remain more photostable than ITIC despite the slight increase in trap-assisted recombination. We can thus conclude that the preserved crystallinity upon illumination is the reason why the PBDB-T:ITIC:[70]PCBM ternary blend film remains the most efficient. Key in this preservation of the crystallinity upon illumination seems to be the incorporation of [70]PCBM in the PBDB-T:ITIC-based blends, that helps to enhance the system's photostability.

\section{Conclusion}

The goal of the current study is to find a way to make the nonfullerene acceptor ITIC-based solar cells more photostable without compromising the power conversion efficiency. This study has shown that this is achievable by means of ternary blends by carefully tuning the ratio of the acceptors in the D:A:A blends to maximise the output of the resulting ternary blend organic solar cells. In our case the ratio of $1: 0.7: 0.3$ of PBDB-T: ITIC: [70]PCBM blend helped in achieving our goal. Thus, the resulting ternary blend solar cells are more photostable over the period of study than the two binary solar cells, especially the PBDB-T:ITIC binary cell, while keeping a higher efficiency than both. The finding of the photostability of the ternary blend solar cells, in this particular case, is also not only valid for the ratios of $1: 0.8: 0.2$ and $1: 0.9: 0.1$ but also for a different donor polymer, PTB7-Th. The ternary blend resilience to photodegradation is explained by the fact that they retain more their crystallinity and molecular packing structure over time compared to the binary blends, especially the ITIC binary organic solar cells. These findings have significant implications for the understanding of the photodegradation mechanisms in [70]PCBM and ITIC-based solar cells and suggest that a better understanding into the ternary blend solar cells could pave a way to more photostable devices.

\section{Experimental methods}

Materials

All materials used are commercially available: PBDB-T and ITIC were purchased from Solarmer Energy Inc., while [70]PCBM was supplied by Solenne BV. All the solvents and anhydrous zinc acetate $\left(\mathrm{Zn}(\mathrm{OAc})_{2}\right)$ were obtained from Sigma-Aldrich and used without further purification.

\section{Solutions preparation}

For the polymer:ITIC and polymer:[70]PCBM solutions, blends in a ratio of $1: 1$ are dissolved in anhydrous chlorobenzene (CB) at a concentration of $20 \mathrm{mg} \mathrm{mL}^{-1}$ and stirred at $40{ }^{\circ} \mathrm{C}$ overnight in a glovebox. The same concentrations are used for the ternary devices, by varying the acceptor ratio $\left(\mathrm{A}_{1}: \mathrm{A}_{2}\right)$ from 0 to 1 . The ratio was varied between $1: 1$ to the best known $1: 1.5$ as used before for PBDT-TT polymer with [70] $\mathrm{PCBM}^{34,35}$ when PTB7-Th is used.

Zinc oxide solution was prepared by dissolving zinc acetate (109.67 $\mathrm{mg})$ in 2-methoxyethanol $(1 \mathrm{~mL})$ and ethanolamine $(30.2 \mu \mathrm{L})$. The solution was then stirred at room temperature for a few hours.

\section{Device fabrication}

The following steps of the device fabrication were performed in a cleanroom environment. All devices were prepared on prepatterned ITO glass substrates. All substrates were carefully and successively cleaned in soap, ultraclean water, acetone, and isopropanol (IPA) with sonication for 10 minutes, then spindried. Additional drying steps were carried out in an oven at $140{ }^{\circ} \mathrm{C}$ for 10 minutes and in UV-ozone steriliser for 20 minutes. For conventional OSCs, PEDOT:PSS layer (50 nm) was spincoated on the ITO substrate in ambient and annealed at $140{ }^{\circ} \mathrm{C}$ for 10 minutes to remove the excess water. For inverted devices, zinc oxide solution was spin-coated instead, at $4000 \mathrm{rpm}$ for 40 seconds then annealed at $170{ }^{\circ} \mathrm{C}$ for 30 minutes in air. Then substrates were transferred into a glovebox under inert conditions for the active layer spin-coating. Spin-coating was performed at $1500 \mathrm{rpm}$ for 5 seconds, followed by active spindrying at $400 \mathrm{rpm}$ for 60 seconds to reach a thickness of $100 \mathrm{~nm}$. Finally, the top electrodes were thermally deposited in an evaporator under vacuum at $<1 \times 10^{-7} \operatorname{mbar}$ : $\operatorname{LiF}(1 \mathrm{~nm})$ and $\mathrm{Al}(100 \mathrm{~nm})$ for conventional configuration, or $\mathrm{MoO}_{3}$ $(10 \mathrm{~nm})$ and $\mathrm{Al}$ or $\mathrm{Ag}(100 \mathrm{~nm})$ for inverted devices.

\section{Characterisations}

UV-visible absorption. UV-visible profiles were obtained from a Shimadzu UV-VIS-NIR spectrophotometer (UV-3600), with tungsten-iodide (WI) monochromatic light source in a range of $300 \mathrm{~nm}$ to $900 \mathrm{~nm}$. Glass or ITO substrate absorption profiles were taken first as references, and corrected for subsequently.

Morphology \& thickness measurements. Atomic force microscopy (AFM) measurements were obtained using a Bruker Nanoscope V AFM in Scan Asyst mode. The layer thicknesses were measured using a Bruker Dektak XT profilometer.

2D grazing incidence wide-angle X-ray scattering (GIWAXS). GIWAXS measurements were performed using the MINA instrument, an X-ray scattering instrument built on a $\mathrm{Cu}$ rotating anode source $(l=1.5413 \AA)$. 2D patterns were collected using a Vantec500 detector $(1024 \times 1024$ pixel array with a pixel size 
$136 \times 136$ microns) located $122 \mathrm{~mm}$ away from the sample. The thin films were placed in reflection geometry at certain incident angles $a_{\mathrm{i}}$ with respect to the direct beam using a Huber goniometer. GIWAXS patterns were acquired using incident angles of $0.2^{\circ}$ (close to the incident angle of the materials). An exposure time of $1 \mathrm{~h}$ per pattern was used. The direct beam centre position on the detector and the sample-to-detector distance were calibrated using the diffraction rings from standard silver behenate and $\mathrm{Al}_{2} \mathrm{O}_{3}$ powders.

\section{Conflicts of interest}

The authors declare no competing interests.

\section{Acknowledgements}

The work is funded by the Zernike Bonus Incentive Scheme Grant. This is a publication by the FOM Focus Group "Next Generation Organic Photovoltaics", participating in the Dutch Institute for Fundamental Energy Research (DIFFER). N. Y. D. would like to acknowledge A. Kamp and T. Zaharia for technical support.

\section{References}

1 J.-H. Kim, A. Gadisa, C. Schaefer, H. Yao, B. R. Gautam, N. Balar, M. Ghasemi, I. Constantinou, F. So, B. T. O’Connor, K. Gundogdu, J. Hou and H. Ade, J. Mater. Chem. A, 2017, 5, 13176-13188.

2 D. Baran, R. S. Ashraf, D. A. Hanifi, M. Abdelsamie, N. Gasparini, J. A. Röhr, S. Holliday, A. Wadsworth, S. Lockett, M. Neophytou, C. J. M. Emmott, J. Nelson, C. J. Brabec, A. Amassian, A. Salleo, T. Kirchartz, J. R. Durrant and I. McCulloch, Nat. Mater., 2017, 16, 363-369.

3 R. Yu, S. Zhang, H. Yao, B. Guo, S. Li, H. Zhang, M. Zhang and J. Hou, Adv. Mater., 2017, 29, 1-6.

4 W. Zhong, J. Cui, B. Fan, L. Ying, Y. Wang, X. Wang, G. Zhang, X. F. Jiang, F. Huang and Y. Cao, Chem. Mater., 2017, 29, 8177-8186.

5 H. Lu, J. Zhang, J. Chen, Q. Liu, X. Gong, S. Feng, X. Xu, W. Ma and Z. Bo, Adv. Mater., 2016, 28, 9559-9566.

6 Y. J. Hwang, H. Li, B. A. E. Courtright, S. Subramaniyan and S. A. Jenekhe, Adv. Mater., 2016, 28, 124-131.

7 L. Zhong, L. Gao, H. Bin, Q. Hu, Z. G. Zhang, F. Liu, T. P. Russell, Z. Zhang and Y. Li, Adv. Energy Mater., 2017, 7, $1-7$.

8 J. W. Lee, Y. S. Choi, H. Ahn and W. H. Jo, ACS Appl. Mater. Interfaces, 2016, 8, 10961-10967.

9 N. Gasparini, X. Jiao, T. Heumueller, D. Baran, G. J. Matt, S. Fladischer, E. Spiecker, H. Ade, C. J. Brabec and T. Ameri, Nat. Energy, 2016, 1, 16118.

10 N. Gasparini, L. Lucera, M. Salvador, M. Prosa, G. D. Spyropoulos, P. Kubis, H. J. Egelhaaf, C. J. Brabec and T. Ameri, Energy Environ. Sci., 2017, 10, 885-892.
11 P. Cheng, J. Wang, Q. Zhang, W. Huang, J. Zhu, R. Wang, S. Y. Chang, P. Sun, L. Meng, H. Zhao, H. W. Cheng, T. Huang, Y. Liu, C. Wang, C. Zhu, W. You, X. Zhan and Y. Yang, Adv. Mater., 2018, 30, 1-8.

12 Z. Xiao, X. Jia and L. Ding, Sci. Bull., 2017, 62, 1562-1564.

13 Y. Xie, F. Yang, Y. Li, M. A. Uddin, P. Bi, B. Fan, Y. Cai, X. Hao, H. Y. Woo, W. Li, F. Liu and Y. Sun, Adv. Mater., 2018, 1803045.

14 T. Kumari, S. M. Lee, S. H. Kang, S. Chen and C. Yang, Energy Environ. Sci., 2017, 10, 258-265.

15 L. Meng, Y. Zhang, X. Wan, C. Li, X. Zhang, Y. Wang, X. Ke, Z. Xiao, L. Ding, R. Xia, H. Yip, Y. Cao and Y. Chen, Science, 2018, 2612, 1-10.

16 J. You, L. Dou, Z. Hong, G. Li and Y. Yang, Prog. Polym. Sci., 2013, 38, 1909-1928.

17 L. Lu, M. A. Kelly, W. You and L. Yu, Nat. Photonics, 2015, 9, 491-500.

18 M. Campoy-Quiles, Y. Kanai, A. El-Basaty, H. Sakai and H. Murata, Org. Electron., 2009, 10, 1120-1132.

19 C. Kästner, S. Rathgeber, D. A. M. Egbe and H. Hoppe, J. Mater. Chem. A, 2013, 1, 3961.

20 R. Bechara, N. Leclerc, P. Lévêque, F. Richard, T. Heiser and G. Hadziioannou, Appl. Phys. Lett., 2008, 93, 013306.

21 A. D. de Zerio and C. Müller, Adv. Energy Mater., 2018, 1702741, 1-18.

22 T. Liu, Y. Guo, Y. Yi, L. Huo, X. Xue, X. Sun, H. Fu, W. Xiong, D. Meng, Z. Wang, F. Liu, T. P. Russell and Y. Sun, Adv. Mater., 2016, 28, 10008-10015.

23 R. A. Street, D. Davies, P. P. Khlyabich, B. Burkhart and B. C. Thompson, J. Am. Chem. Soc., 2013, 135, 986-989.

24 P. P. Khlyabich, B. Burkhart and B. C. Thompson, J. Am. Chem. Soc., 2012, 134, 9074-9077.

25 H. Cha, S. Wheeler, S. Holliday, S. D. Dimitrov, A. Wadsworth, H. H. Lee, D. Baran, I. McCulloch and J. R. Durrant, Adv. Funct. Mater., 2018, 28, 1704389.

26 H. Li, Y.-J. Hwang, B. A. E. Courtright, F. N. Eberle, S. Subramaniyan and S. A. Jenekhe, Adv. Mater., 2015, 27, 3266-3272.

27 N. Gasparini, M. Salvador, T. Heumueller, M. Richter, A. Classen, S. Shrestha, G. J. Matt, S. Holliday, S. Strohm, H.-J. Egelhaaf, A. Wadsworth, D. Baran, I. McCulloch and C. J. Brabec, Adv. Energy Mater., 2017, 7, 1701561.

28 Y. Wang, M. J. Jafari, N. Wang, D. Qian, F. Zhang, T. Ederth, E. Moons, J. Wang, O. Inganäs, W. Huang and F. Gao, J. Mater. Chem. A, 2018, 6, 11884-11889.

29 N. Y. Doumon, M. V. Dryzhov, F. V. Houard, V. M. Le Corre, A. Rahimi Chatri, P. Christodoulis and L. J. A. Koster, ACS Appl. Mater. Interfaces, 2019, DOI: 10.1021/acsami. 8 b20493.

30 D. Baran, N. Gasparini, A. Wadsworth, C. H. Tan, N. Wehbe, X. Song, Z. Hamid, W. Zhang, M. Neophytou, T. Kirchartz, C. J. Brabec, J. R. Durrant and I. Mcculloch, Nat. Commun., 2018, 9, 2059.

31 H. Cha, J. Wu, A. Wadsworth, J. Nagitta, S. Limbu, S. Pont, Z. Li, J. Searle, M. F. Wyatt, D. Baran, J. S. Kim, I. McCulloch and J. R. Durrant, Adv. Mater., 2017, 29, 1-8. 
32 N. Gasparini, M. Salvador, S. Strohm, T. Heumueller, I. Levchuk, A. Wadsworth, J. H. Bannock, J. C. de Mello, H. J. Egelhaaf, D. Baran, I. Mcculloch and C. J. Brabec, Adv. Energy Mater., 2017, 1700770, 1-7.

33 S. H. Liao, H. J. Jhuo, Y. S. Cheng and S. A. Chen, Adv. Mater., 2013, 25, 4766-4771.
34 N. Y. Doumon, G. Wang, R. C. Chiechi and L. J. A. Koster, J. Mater. Chem. C, 2017, 5, 6611-6619.

35 N. Y. Doumon and L. J. A. Koster, Sol. RRL, 2019, 1800301.

36 B. Wang, Y. Fu, C. Yan, R. Zhang, Q. Yang, Y. Han and Z. Xie, Front. Chem., 2018, 6, 1-8. 